\title{
BMJ Open Analysis of multimorbidity networks associated with different factors in Northeast China: a cross- sectional analysis
}

\author{
Jianxing Yu, Yingying Li, Zhou Zheng, Huanhuan Jia (D) , Peng Cao, \\ Yuzhen Qiangba, Xihe Yu
}

To cite: Yu J, Li Y, Zheng Z, et al. Analysis of multimorbidity networks associated with different factors in Northeast China: a cross-sectional analysis. BMJ Open 2021;11:e051050. doi:10.1136/ bmjopen-2021-051050

- Prepublication history for this paper is available online. To view these files, please visit the journal online (http://dx.doi. org/10.1136/bmjopen-2021 051050).

Received 13 March 2021 Accepted 18 0ctober 202

\section{Check for updates}

(C) Author(s) (or their employer(s)) 2021. Re-use permitted under CC BY-NC. No commercial re-use. See rights and permissions. Published by BMJ.

Social Medicine and Health Service Management, School of Public Health, Jilin University, Changchun, China

Correspondence to Dr Xihe Yu; xhyu@jlu.edu.cn

\section{ABSTRACT}

Objectives This study aimed to identify and study the associations and co-occurrence of multimorbidity, and assessed the associations of diseases with sex, age and hospitalisation duration.

Design Cross-sectional.

Setting 15 general hospitals in Jilin Province, China. Participants A total of 431295 inpatients were enrolled through a cross-sectional study in Jilin Province, China.

Primary outcome measures The complex relationships of multimorbidity were presented as weighted networks.

Results The distributions of the numbers of diseases differed significantly by sex, age and hospitalisation duration $(p<0.001)$. Cerebrovascular diseases $(C D)$, hypertensive diseases $(\mathrm{HyD})$, ischaemic heart diseases (IHD) and other forms of heart disease (OFHD) showed the highest weights in the multimorbidity networks. The connections between different sexes or hospitalisation duration and diseases were similar, while those between different age groups and diseases were different.

Conclusions CD, HyD, IHD and OFHD were the central points of disease clusters and directly or indirectly related to other diseases or factors. Thus, effective interventions for these diseases should be adopted. Furthermore, different intervention strategies should be developed according to multimorbidity patterns in different age groups.

\section{INTRODUCTION}

The term multimorbidity broadly refers to the presence of two or more health conditions (diseases) in a single individual. ${ }^{1-4}$ With the continuing increase in life expectancy, multimorbidity has become a worldwide public health issue as it increases with age. ${ }^{5}$ Additionally, multimorbidity is associated with increased adverse health outcomes such as poor quality of life, disability, hospitalisation, mortality and the concomitant use of healthcare resources and expenditure..$^{6-9}$ Furthermore, multimorbidity is also costly for both individuals and the healthcare system, with healthcare utilisation and costs increasing with each additional condition, ${ }^{10-12}$
Strengths and limitations of this study

- This study visually demonstrated the differences in multimorbidity according to sex, age group and hospitalisation duration.

- Adjusting the analysis of multimorbidity patterns to the individual level rather than disease level could identify and study the associations and cooccurrence of multimorbidity.

- The model can be applied to assess the patterns of multimorbidity associated with different factors, and provide meaningful information for clinicians.

- The results were based on a cross-sectional study in Jilin Province, China, which might limit the generalisability of the results.

particularly in China, the world's most populous country. ${ }^{1314}$ Therefore, identifying the associations and co-occurrence of multimorbidity is an essential public health issue that requires urgent attention.

Most of the published literature on multimorbidity patterns focuses on disease level rather than individual level. ${ }^{1} 1015$ Adjusting the analysis of multimorbidity patterns to the individual level rather than disease level could identify and study the associations and co-occurrence of multimorbidity. Studying and treating diseases in isolation may not only lead to inefficiencies and duplication in the case of multimorbid patients but may also have serious implications if treatment for one disease contradicts that for another. ${ }^{1}$ A multidimensional approach is required to understand the patterns of multimorbidity and recognise the associations between conditions within these patterns.

Furthermore, Aguado et $a l^{16}$ pointed out that multimorbidity is a complex phenomenon that can be assessed using network analysis. Moreover, research on multiple disease networks has attracted increasing 
attention in recent years. In their analysis of phenotyping networks, Hidalgo et $a l^{17}$ reported that patients with highly connected diseases tended to die sooner. Glicksberg $e t a l^{18}$ observed race-specific disease networks based on a large-scale analysis of electronic medical records. In their multimorbidity network analyses, Kalgotra et al identified specific differences in disease diagnosis by sex and proposed questions for behavioural, clinical, biological and policy research; these researchers also identified specific differences in diagnoses among different population groups. ${ }^{190}$

However, these studies, while powerful and groundbreaking, did not adequately address the question of the associations of age, hospitalisation duration and diseases in their multimorbidity networks due to the limitations of their database. Thus, the present study aimed to identify and study the associations and co-occurrence of multimorbidity and provide meaningful information for clinicians. Another objective was to better understand the associations between common health conditions (diseases) to advance research into the mechanisms underpinning these common health conditions (diseases) associations. Finally, this study also assessed the associations of diseases with sex, age and hospitalisation duration.

\section{METHODS}

\section{Study population}

This study analysed data obtained from the hospital information systems or electronic medical record systems of 15 general hospitals in Jilin Province, China. The research objects were inpatients between 1 January 2018 and 31 December 2018, and the final study comprised 516399 inpatients. For each included inpatient, the extracted variables were sex, age, hospitalisation duration, disease names and International Statistical Classification of Diseases and Related Health Problems 10th Revision (ICD-10) classifications. To ensure the effectiveness and representativeness of the constructed disease network, the original medical data set was preprocessed to eliminate invalid patient records, including the following: (1) inpatients with some conditions or diseases such as injury, poisoning, certain infectious or parasitic diseases and congenital malformations; and (2) diseases occurring in fewer than 1000 inpatients (as the disease network was constructed without considering rare diseases). Finally, this study included 72 diseases to explore multimorbidity in 431295 inpatients.

\section{Data collection}

A multistage stratified random sampling method was used to obtain the sample data. First, through a comprehensive assessment of the geographical location, economic level and health service status of each city, Jilin, Changchun, Baicheng, Yanbian and Tonghua were finally included in the sample area, three general hospitals from each of these locations were then random selected. Finally, based on the administrative division, 15 general hospitals were selected as the monitoring institutions for this study. The inpatient data comprised continuous medical records, including indicators such as sex, age, hospitalisation duration, ICD-10 classification and disease name. To improve the data accuracy, we recruited and trained 20 people with proficiency in Excel software and medical backgrounds to form a professional team to check the accuracy of the basic information. Thirty general practitioners with more than 3 years of work experience confirmed that the names of the diseases matched the ICD-10 classifications.

\section{Statistical analysis}

The categorical variables in this study were expressed as counts and percentages. Rao-Scott- $\chi^{2}$ tests were used to compare the distributions of the numbers of diseases and the complex relationships of multimorbidity were presented as weighted networks. The nodes represented diseases/factors, with the sizes of the nodes indicating their weight relative to all other diseases/factors. The edges represented the co-occurrence of a multimorbidity pair in the network, with the weight of the edge proportional to the prevalence of each pair. For inpatients with more than two diseases, the count of each multimorbidity pair would have an increment of 1 (eg, for an inpatient with ischaemic heart diseases (IHD), hypertensive disease $(\mathrm{HyD})$ and cerebrovascular disease $(\mathrm{CD})$, the multimorbidity pairs of IHD\&HyD, IHD\&CD and HyD\&CD would increment by 1 ). The degree was defined as the number of nodes to which a focus node was connected, and was used to measure the node's participation in the network. The sparsity of the network was evaluated using the network density and average degree. The network density of an undirected graph with $\mathrm{M}$ edges and $\mathrm{N}$ nodes was defined as $2 \mathrm{M} / \mathrm{N}(\mathrm{N}-1)$, which described the proportion of potential connections $(\mathrm{N}(\mathrm{N}-1) / 2)$ in a network with actual connections (M). The larger the network density (or average degree), the denser the network. ${ }^{21-23}$ The networks were analysed using the $\mathrm{R}$ package igraph. All statistical analyses were performed using R V.3.6.1 (R Core Team (2014). R: A language and environment for statistical computing. R Foundation for Statistical Computing, Vienna, Austria. URL http://www.R-project.org/). Statistical significance was set at $\mathrm{p}<0.05$.

\section{Patient and public involvement}

Patients were not involved in the study based on anonymised data.

\section{RESULTS}

We analysed data from 431295 inpatients in Jilin Province, China. As shown in table 1, the distributions of the numbers of diseases differed significantly by sex, age and hospitalisation duration $(\mathrm{p}<0.001)$. Additionally, the number of male inpatients was higher than that of female inpatients and the proportion of male inpatients with 2 or $\geq 3$ diseases was higher than that of female inpatients. Further, the proportion of inpatients with one disease 
Table 1 Descriptive characteristics of the inpatients according to the number of diseases

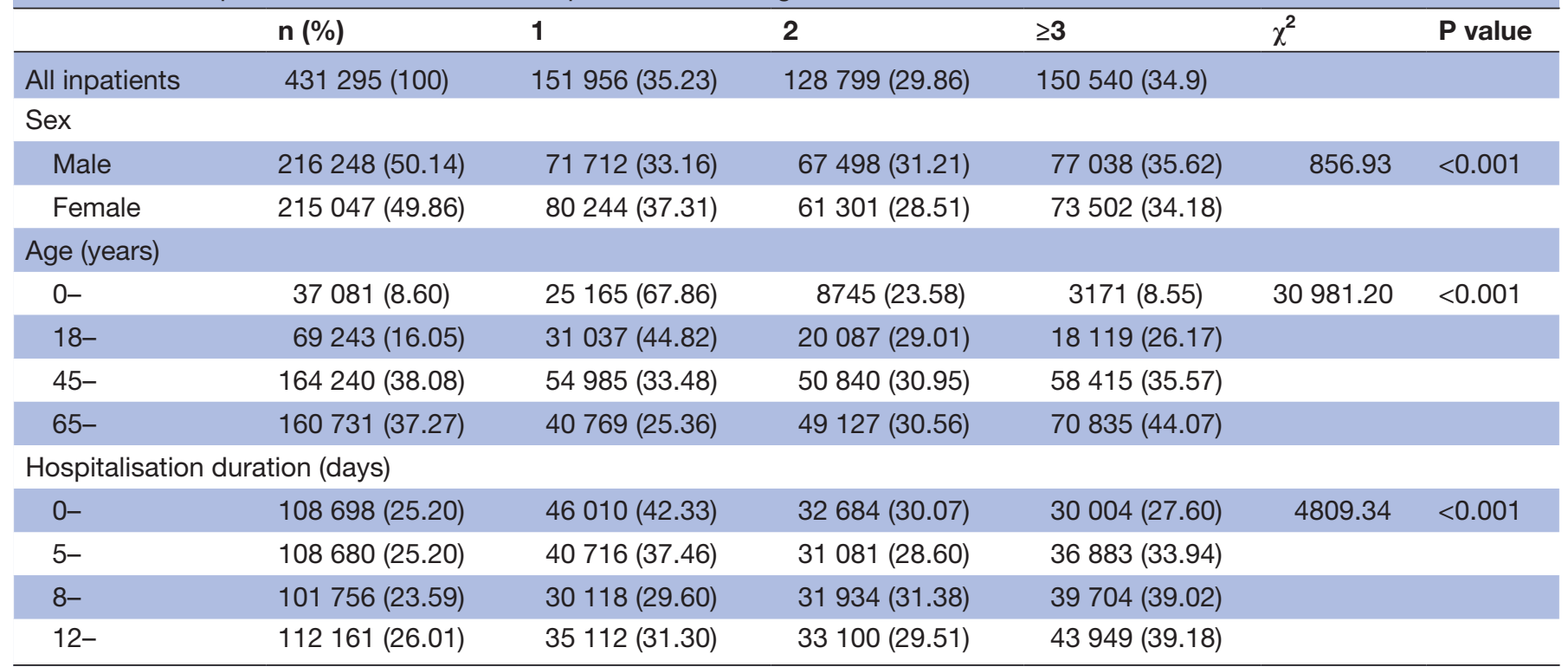

decreased with age, while the proportion of inpatients with $\geq 3$ diseases increased. Moreover, the results of hospitalisation duration were similar to those for the age groups and the proportion of inpatients with $\geq 3$ diseases increased with increasing hospitalisation duration.

Table 2 shows the abbreviations and proportions of the 72 diseases. Among the 72 diseases, the maximum and minimum percentages were 19.34 and 0.30 , respectively. Additionally, IHD, HyD, CD, other forms of heart disease (OFHD) and malignant neoplasms (MNE) had frequencies exceeding 50000.

Figure 1 shows a visual representation of the network according to sex, age and hospitalisation duration. Both sexes had notably more connections in the 45-64 years and 65- years age groups; however, female inpatients had more connections for the 18-44 years age group compared with that in male inpatients. Additionally, the 45-64 years and 65- years age groups had notably more connections with the hospitalisation duration, with the 65- years age group showing more connections to 12days hospitalisation duration. Finally, the connections between different sexes and hospitalisation duration were similar.

Figure 2 shows the multimorbidity network for 72 diseases. CD, HyD, IHD and OFHD had notably high weights (illustrated by the sizes of the nodes). In addition, the 'IHD-OFHD-HyD' triangle and 'CD-HyD' exhibited notably high connections in the multimorbidity networks (illustrated by the thicknesses of the lines connected to these nodes). Although MNE had notably high weights, no high connections to other diseases were observed compared with those for other high-weight diseases.

As illustrated in figure 3, the 'IHD-OFHD-Male', 'IHDOFHD-Female', 'CD-HyD-Male' and 'CD-HyD-Female' triangles showed notably high connections in the networks. Moreover, CD and diabetes mellitus (DME) had more notable connections with male inpatients compared with those in female inpatients.

Figure 4 shows the connections between different age groups and the 72 diseases. The older age group not only had more inpatients (illustrated by the sizes of the nodes) but also had more connections with other diseases (illustrated by the thicknesses of the lines connected to these nodes). Compared with other diseases, acute upper respiratory infections (AURI), influenza and pneumonia (IP) and OFHD had notably more connections with the $<18$ years age group. However, the $18-44$ years age group had notably more connections to complications of labour and delivery (CLD), delivery (Del), maternal care related to the fetus and amniotic cavity and possible delivery problems (MCFAP), other maternal disorders predominantly related to pregnancy (OMDP) and metabolic disorders $(\mathrm{MeD})$, and four of which were related to pregnancy and delivery. CD, HyD, IHD, OFHD and DME had notably more connections in the 45-64 years age group, and mainly cardiovascular and cerebrovascular diseases. In the 65- years age group, the 'CD-HyD-65-' and 'IHD-OFHD-65-' triangles showed the most network connections.

Figure 5 shows the networks for the different groups of hospitalisation duration and diseases. The weights were similar for the different groups of hospitalisation duration (illustrated by the node sizes). Moreover, CD, IHD, HyD, OFHD had more connections with the different groups of hospitalisation duration (illustrated by the thicknesses of the lines connected to these nodes).

\section{DISCUSSION}

A major strength of this study was that it used a largescale, real-world clinical database of 431295 inpatients in Jilin province, China. Additionally, the patterns of 
Table 2 Abbreviations and proportions of the 72 diseases

\section{ICD-10}

\begin{tabular}{|c|c|c|c|c|c|}
\hline No & $\begin{array}{l}\text { ICD-10 } \\
\text { code range }\end{array}$ & Disease name & Abbreviation & Frequency & $\begin{array}{l}\text { Percentage } \\
(\%)\end{array}$ \\
\hline 1 & D60-D64 & Aplastic and other anaemias & $\mathrm{AOA}$ & 12938 & 3.00 \\
\hline 2 & M00-M25 & Arthropathies & Arth & 8937 & 2.07 \\
\hline 3 & J00-J06 & Acute upper respiratory infections & AURI & 9014 & 2.09 \\
\hline 4 & D10-D36 & Benign neoplasms & BNE & 15462 & 3.59 \\
\hline 5 & $160-169$ & Cerebrovascular diseases & $C D$ & 68144 & 15.80 \\
\hline 6 & D80-D89 & Certain disorders involving the immune mechanism & $\mathrm{CIM}$ & 1299 & 0.30 \\
\hline 7 & O60-075 & Complications of labour and delivery & CLD & 6667 & 1.55 \\
\hline 8 & J40-J47 & Chronic lower respiratory diseases & CLRD & 15772 & 3.66 \\
\hline 9 & D65-D69 & $\begin{array}{l}\text { Coagulation defects, purpura and other haemorrhagic } \\
\text { conditions }\end{array}$ & $\mathrm{CPH}$ & 2432 & 0.56 \\
\hline 10 & G80-G83 & Cerebral palsy and other paralytic syndromes & CPPS & 1819 & 0.42 \\
\hline 11 & $170-179$ & Diseases of arteries, arterioles and capillaries & DAAC & 8961 & 2.08 \\
\hline 12 & H30-H36 & Disorders of choroid and retina & DCR & 4609 & 1.07 \\
\hline 13 & L20-L30 & Dermatitis and eczema & DE & 1575 & 0.37 \\
\hline 14 & O80-084 & Delivery & Del & 7782 & 1.80 \\
\hline 15 & K80-K87 & Disorders of gallbladder, biliary tract and pancreas & DGBP & 16296 & 3.78 \\
\hline 16 & $\mathrm{H} 25-\mathrm{H} 28$ & Disorders of lens & Dle & 11856 & 2.75 \\
\hline 17 & E10-E14 & Diabetes mellitus & DME & 46510 & 10.78 \\
\hline 18 & N40-N51 & Diseases of male genital organs & DMGO & 6543 & 1.52 \\
\hline 19 & K35-K38 & Diseases of appendix & DOA & 4839 & 1.12 \\
\hline 20 & K70-K77 & Diseases of liver & $\mathrm{DOL}$ & 26663 & 6.18 \\
\hline 21 & K65-K67 & Diseases of peritoneum & DOP & 2356 & 0.55 \\
\hline 22 & M40-M54 & Dorsopathies & Dors & 10131 & 2.35 \\
\hline 23 & K20-K31 & Diseases of oesophagus, stomach and duodenum & DOSD & 21127 & 4.90 \\
\hline 24 & K00-K14 & Diseases of oral cavity, salivary glands and jaws & DOSJ & 1803 & 0.42 \\
\hline 25 & E00-E07 & Disorders of thyroid gland & DTG & 7550 & 1.75 \\
\hline 26 & $\mathrm{H} 43-\mathrm{H} 45$ & Disorders of vitreous body and globe & DVBG & 1387 & 0.32 \\
\hline 27 & $180-189$ & $\begin{array}{l}\text { Diseases of veins, lymphatic vessels and lymph } \\
\text { nodes, not elsewhere classified }\end{array}$ & DVLL & 7191 & 1.67 \\
\hline 28 & G40-G47 & Episodic and paroxysmal disorders & EPD & 15619 & 3.62 \\
\hline 29 & N00-N08 & Glomerular diseases & GD & 3234 & 0.75 \\
\hline 30 & $\mathrm{H} 40-\mathrm{H} 42$ & Glaucoma & Gla & 1962 & 0.45 \\
\hline 31 & K40-K46 & Hernia & $\mathrm{HI}$ & 3516 & 0.82 \\
\hline 32 & $|10-| 15$ & Hypertensive diseases & HyD & 78747 & 18.26 \\
\hline 33 & N70-N77 & Inflammatory diseases of female pelvic organs & IDFP & 3014 & 0.70 \\
\hline 34 & I20-|25 & Ischaemic heart diseases & IHD & 83428 & 19.34 \\
\hline 35 & J09-J18 & Influenza and pneumonia & IP & 37290 & 8.65 \\
\hline 36 & L00-L08 & Infections of the skin and subcutaneous tissue & ISST & 2403 & 0.56 \\
\hline 37 & J60-J70 & Lung diseases due to external agents & LDEA & 1328 & 0.31 \\
\hline 38 & O30-048 & $\begin{array}{l}\text { Maternal care related to the fetus and amniotic cavity } \\
\text { and possible delivery problems }\end{array}$ & MCFAP & 8041 & 1.86 \\
\hline 39 & E70-E90 & Metabolic disorders & $\mathrm{MeD}$ & 43477 & 10.08 \\
\hline 40 & $\mathrm{CO0-C97}$ & Malignant neoplasms & MNE & 56980 & 13.21 \\
\hline 41 & D50-D53 & Nutritional anaemias & NAN & 1722 & 0.40 \\
\hline 42 & N80-N98 & Non-inflammatory disorders of female genital tract & NDFG & 10227 & 2.37 \\
\hline
\end{tabular}




\begin{tabular}{|c|c|c|c|c|c|}
\hline No & $\begin{array}{l}\text { ICD-10 } \\
\text { code range }\end{array}$ & Disease name & Abbreviation & Frequency & $\begin{array}{l}\text { Percentage } \\
(\%)\end{array}$ \\
\hline 43 & K50-K52 & Non-infective enteritis and colitis & NEC & 13421 & 3.11 \\
\hline 44 & G50-G59 & Nerve, nerve root and plexus disorders & NPD & 1964 & 0.46 \\
\hline 45 & F40-F48 & Neurotic, stress-related and somatoform disorders & NSS & 1532 & 0.36 \\
\hline 46 & D37-D48 & Neoplasms of uncertain or unknown behaviour & NUB & 3895 & 0.90 \\
\hline 47 & J20-J22 & Other acute lower respiratory infections & OARI & 7348 & 1.70 \\
\hline 48 & $\mathrm{H} 49-\mathrm{H} 52$ & $\begin{array}{l}\text { Disorders of ocular muscles, binocular movement, } \\
\text { accommodation and refraction }\end{array}$ & OBAR & 2967 & 0.69 \\
\hline 49 & D70-D77 & Other diseases of blood and blood-forming organs & ОВо & 3116 & 0.72 \\
\hline 50 & M80-M94 & Osteopathies and chondropathies & $\mathrm{OC}$ & 5715 & 1.33 \\
\hline 51 & G30-G32 & Other degenerative diseases of the nervous system & ODDNS & 2133 & 0.49 \\
\hline 52 & K90-K93 & Other diseases of the digestive system & ODDS & 5737 & 1.33 \\
\hline 53 & K55-K63 & Other diseases of intestines & ODI & 17525 & 4.06 \\
\hline 54 & N25-N29 & Other disorders of kidney and ureter & ODKU & 4442 & 1.03 \\
\hline 55 & G90-G99 & Other disorders of the nervous system & ODNS & 3302 & 0.77 \\
\hline 56 & J90-J94 & Other diseases of pleura & ODP & 5222 & 1.21 \\
\hline 57 & J95-J99 & Other diseases of the respiratory system & ODRS & 13260 & 3.07 \\
\hline 58 & J30-J39 & Other diseases of upper respiratory tract & ODRT & 5612 & 1.30 \\
\hline 59 & N30-N39 & Other diseases of urinary system & ODUS & 9353 & 2.17 \\
\hline 60 & 130-|52 & Other forms of heart disease & OFHD & 67632 & 15.68 \\
\hline 61 & O20-O29 & $\begin{array}{l}\text { Other maternal disorders predominantly related to } \\
\text { pregnancy }\end{array}$ & OMDP & 9398 & 2.18 \\
\hline 62 & 094-099 & Other obstetric conditions, not elsewhere classified & OOC & 3138 & 0.73 \\
\hline 63 & 010-016 & $\begin{array}{l}\text { Oedema, proteinuria and hypertensive disorders in } \\
\text { pregnancy, childbirth and the puerperium }\end{array}$ & OPHD & 1390 & 0.32 \\
\hline 64 & J80-J84 & $\begin{array}{l}\text { Other respiratory diseases principally affecting the } \\
\text { interstitium }\end{array}$ & ORDI & 1922 & 0.45 \\
\hline 65 & O00-008 & Pregnancy with abortive outcome & PAO & 2809 & 0.65 \\
\hline 66 & I26-|28 & $\begin{array}{l}\text { Pulmonary heart disease and diseases of pulmonary } \\
\text { circulation }\end{array}$ & PHPC & 2135 & 0.50 \\
\hline 67 & N17-N19 & Renal failure & RF & 11562 & 2.68 \\
\hline 68 & N10-N16 & Renal tubulo-interstitial diseases & RTD & 2710 & 0.63 \\
\hline 69 & $\mathrm{H} 15-\mathrm{H} 22$ & Disorders of sclera, cornea, iris and ciliary body & SCIC & 1412 & 0.33 \\
\hline 70 & M30-M36 & Systemic connective tissue disorders & SCTD & 2149 & 0.50 \\
\hline 71 & M60-M79 & Soft tissue disorders & STD & 2204 & 0.51 \\
\hline 72 & N20-N23 & Urolithiasis & Uro & 3961 & 0.92 \\
\hline
\end{tabular}

ICD-10, International Statistical Classification of Diseases and Related Health Problems 10th Revision.

multimorbidity were based on the entire eligible sample. Another strength was that individual level rather than disease level was considered as the unit of analysis. ${ }^{15} 24$ This approach permits a more rational and realistic monitoring of participants than cohort studies to analyse multimorbidity patterns over time. ${ }^{25}$ In the syndemics theory proposed by Singer, ${ }^{26}$ the effects of the presence of multiple diseases on a patient's health differ from their individual independent efffects. In other words, the risk of multimorbidity is greater than the sum of single diseases. Furthermore, networks offer a more global picture because they include not only direct connections but also indirect associations, which provides more accurate information about multimorbidity. However, most current studies have compared different racial groups, ${ }^{1718}$ and few have evaluated the associations of disease patterns with sex, age and hospitalisation duration. The present study not only identified and studied the associations and co-occurrence of multimorbidity and provided meaningful information for clinicians but also 

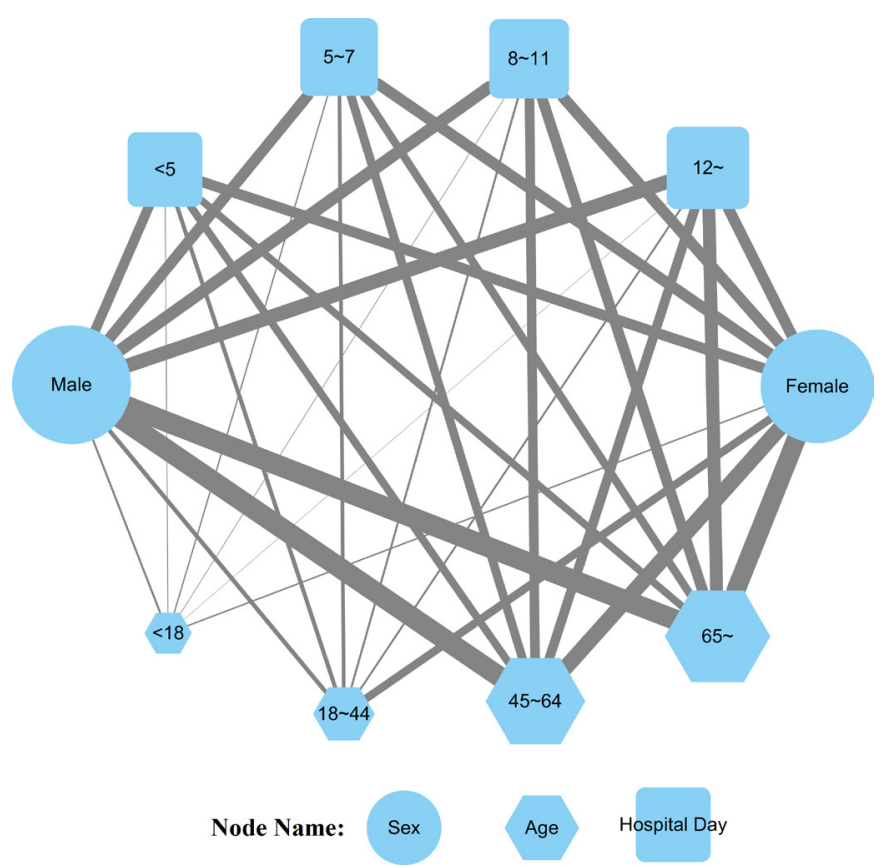

Figure 1 Network of sex, age and hospitalisation duration.

assessed the associations of diseases patterns with sex, age and hospitalisation duration.

Comparing the results of the present study to those by Hidalgo $e a^{17}$ on human phenotype using a dynamic network approach can be used to verify the reliability of the results of this study. Hidalgo et al found that many diseases were associated with HyD or IHD, consistent with the findings of the present study. Hidalgo $e t a l^{17}$ further demonstrated higher comorbidity for DME and HyD in black men compared with white men, and this study also confirmed more notable connections for DME in male inpatients compared with female inpatients.

Previous studies have shown that sex significantly affects multimorbidity. ${ }^{19} 2728$ In the present study, the proportion of multimorbidity was much higher in male inpatients than in female inpatients, a finding consistent with those of other studies. ${ }^{29-31}$ Further, the disease associations in networks of both sexes revealed different disease associations according to sex. In the present study, 'CD-HyD' and 'IHD-OFHD' showed more connections

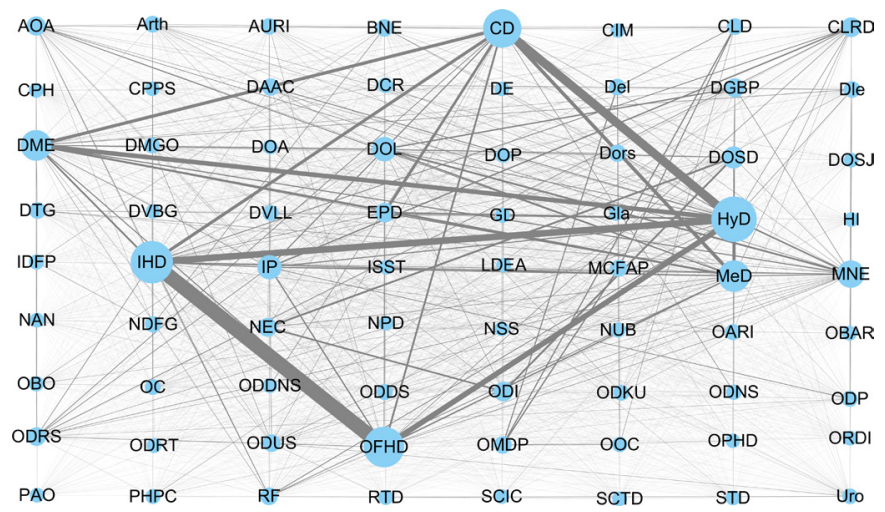

Figure 2 Multimorbidity networks for 72 diseases.

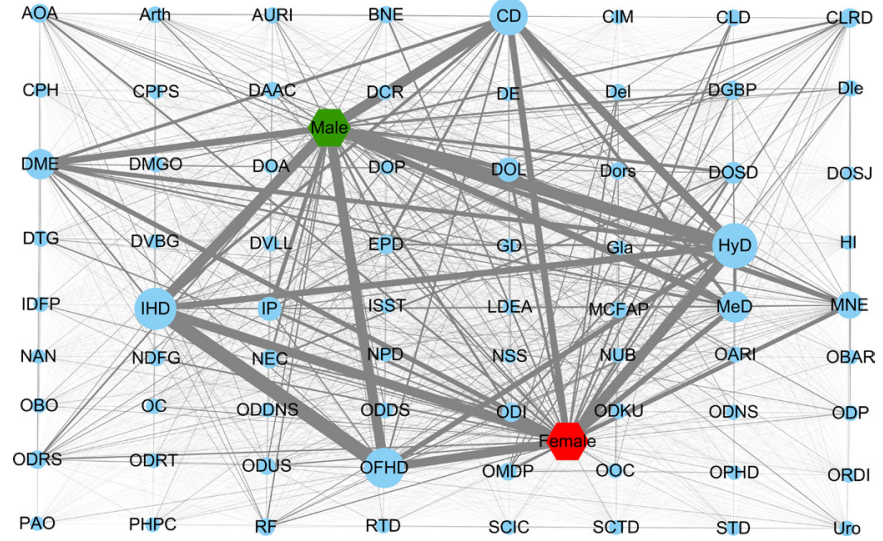

Figure 3 Multimorbidity networks with sex for 72 diseases.

with male inpatients and female inpatients, respectively. The connection between $\mathrm{CD}$ and $\mathrm{HyD}$ in both sexes has been well documented in the literature. ${ }^{32}$ Additionally, $\mathrm{CD}$ and $\mathrm{HyD}$ share common risk factors such as obesity, ${ }^{33}{ }^{34}$ smoking and drinking. ${ }^{35}$ Furthermore, CD and DME showed more connections with male inpatients than female inpatients, also consistent with the findings of other studies. ${ }^{1736} 37$

Multimorbidity is often attributed to the ageing process, with a prevalence of approximately $62 \%$ in individuals aged $65-74$ years and $81.5 \%$ in those older than 85 years of age. ${ }^{38}$ A previous study showed an increasing tendency in the prevalence of multimorbidity in older adults. ${ }^{39} 40$ The present study observed similar results, with more patients in the 65-74 years age group (table 1 and illustrated by the sizes of the nodes in figure 4 ). This may be due to higher body immunity and function in younger people compared with those in older people, thus, the proportion of diseases was lowest in the $0-17$ years age group.

Furthermore, the main diseases differed across age groups; for instance, AURI and IP showed more connections in the $<18$ years age group. Other studies also found similar results. ${ }^{41-43}$ Thus, more attention should be paid to respiratory system diseases in patients $<18$ years. Furthermore, CLD, Del, MCFAP, OMDP and MeD showed notably more connections in the 18-44 years age group.

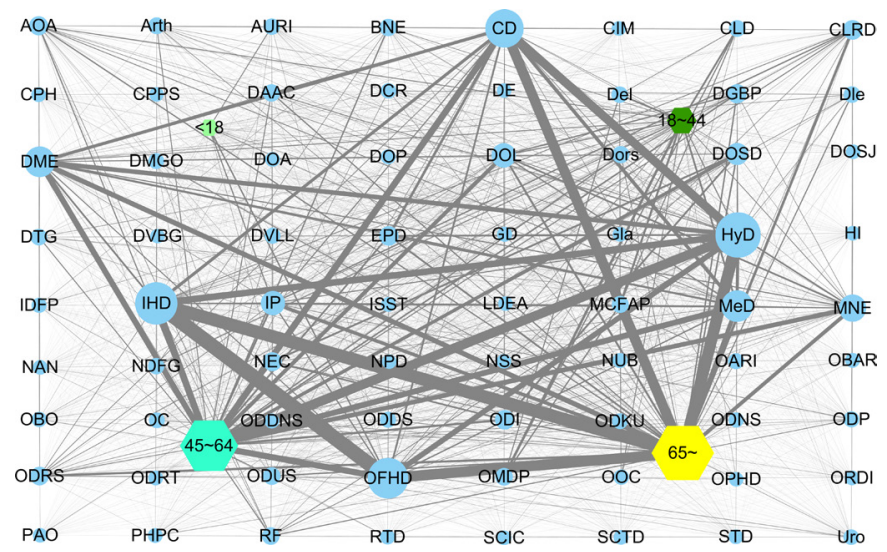

Figure 4 Multimorbidity networks with age for 72 diseases. 


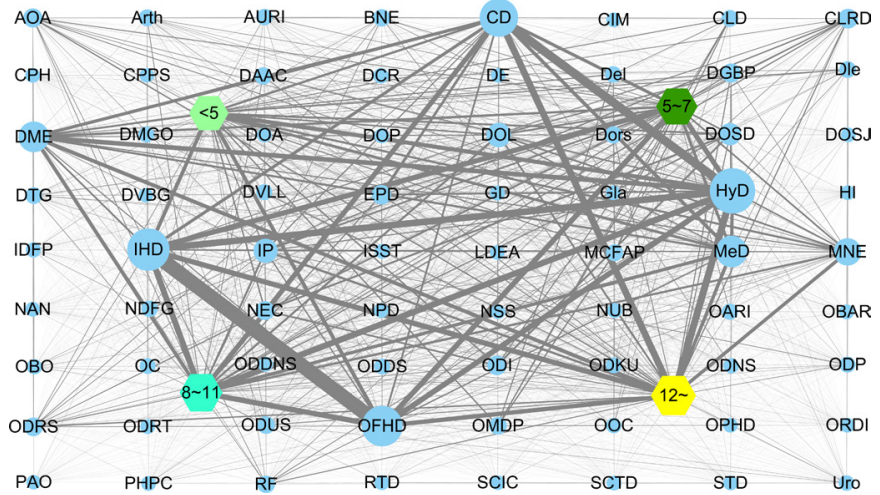

Figure 5 Multimorbidity networks with hospitalisation duration for 72 diseases.

This finding is likely related to the age at which women have children; and other studies have shown that women often develop metabolic disorders during pregnancy. ${ }^{44}$ Thus, more attention should be given to female health. Moreover, the health challenges faced by the 45-64 years age group are more complex than those faced by other age groups because body immunity and function decline with age. Additionally, the population in this age group is under substantial mental stress, leading to feelings of exhaustion and illness. Therefore, multimorbidity studies individuals aged 45-64 years cannot be overlooked, particularly those on CD, HyD, IHD, OFHD and DME. Although this age group had a denser multimorbidity network, the 'CD-HyD-65-' and 'IHD-OFHD-65-' triangles showed more connections than those of other combinations. These findings suggest that different intervention strategies should be developed according to multimorbidity patterns in different age groups.

Finally, the results of this study showed that the stronger the disease connection to other diseases, the stronger the connection to the longer duration of hospitalisation. For example, HyD showed more marked connections with other diseases and a stronger connection with the group of patients with longer hospitalisation duration. Other studies have reported similar results. Specogna $e t$ $a t^{45}$ observed that patients with spontaneous intracerebral haemorrhage arriving at the hospital with $\mathrm{HyD}$ were $31 \%$ more likely to stay in the hospital beyond 1 week per visit compared with non-hypertensive patients.

This study had some limitations. First, the study participants were inpatients in Jilin Province, which could not represent the patterns of multimorbidity in other places. Second, this study investigated only sex, age and hospitalisation duration; however, other factors not considered in this study might have impacted multimorbidity. Finally, diseases occurring in fewer than 1000 inpatients were excluded, which might have caused bias.

\section{CONCLUSION}

The results of this study visually demonstrated the differences in multimorbidity according to sex, age group and hospitalisation duration. Adjusting the analysis of multimorbidity patterns to the individual level revealed that IHD, HyD, CD and OFHD were the central points of disease clusters and were directly or indirectly related to other diseases and factors. Thus, accurate identification and effective intervention for these diseases should be adopted in the healthcare system. Furthermore, the government and relevant departments should develop different intervention strategies according to multimorbidity patterns in different age groups. Finally, the multimorbidity patterns were more consistent with clinical practice, allowing the effective management of patients with multimorbidity.

Acknowledgements We express our gratitude to the participants and colleagues who were involved in the study. We are also appreciative for the support of our funders

Contributors JY and $X Y$ had the original idea for the study and carried out the design. JY and XY provided valuable insight regarding the methodological approach and organization of the manuscript. JY, YL and ZZ carried out the statistical analysis and reviewed the consistency of data included in the paper. JY and YL drafted the manuscript. JY, HJ, PC and YQ revised the manuscript. All authors read and approved the final manuscript.

Funding This study is funding by the Ministry of Education (MOE) in China Project of Humanities and Social Sciences (Project No. 18YJAZH118).

Competing interests None declared.

Patient and public involvement Patients and/or the public were not involved in the design, or conduct, or reporting, or dissemination plans of this research.

\section{Patient consent for publication Not applicable.}

Ethics approval This study was approved by the ethics committee of the School of Public Health, Jilin University (Reference Number: 2018-07-06).

Provenance and peer review Not commissioned; externally peer reviewed.

Data availability statement Data may be obtained from a third party and are not publicly available. These data were from a survey conducted by the School of Public Health, Jilin University in Jilin Province in 2018. Because of relevant regulations, the data cannot be shared.

Open access This is an open access article distributed in accordance with the Creative Commons Attribution Non Commercial (CC BY-NC 4.0) license, which permits others to distribute, remix, adapt, build upon this work non-commercially, and license their derivative works on different terms, provided the original work is properly cited, appropriate credit is given, any changes made indicated, and the use is non-commercial. See: http://creativecommons.org/licenses/by-nc/4.0/.

\section{ORCID iDs}

Huanhuan Jia http://orcid.org/0000-0003-4151-0915

Xihe Yu http://orcid.org/0000-0002-7076-1062

\section{REFERENCES}

1 Barnett K, Mercer SW, Norbury M, et al. Epidemiology of multimorbidity and implications for health care, research, and medical education: a cross-sectional study. Lancet 2012;380:37-43.

2 Tinetti ME, Fried TR, Boyd CM. Designing health care for the most common chronic Condition-Multimorbidity. JAMA 2012;307:2493-4.

3 Ng SK, Tawiah R, Sawyer M, et al. Patterns of multimorbid health conditions: a systematic review of analytical methods and comparison analysis. Int J Epidemiol 2018;47:1687-704.

4 Van den Akker M, Buntinx F, Knottnerus JA. Comorbidity or multimorbidity: what's in a name? A review of literature. The European Journal of General Practice 1996;2:65-70.

5 Salisbury C, Johnson L, Purdy S, et al. Epidemiology and impact of multimorbidity in primary care: a retrospective cohort study. $\mathrm{Br} J$ Gen Pract 2011;61:e12-21.

6 Bähler C, Huber CA, Brüngger B, et al. Multimorbidity, health care utilization and costs in an elderly community-dwelling population: 
a claims data based observational study. BMC Health Serv Res 2015;15:23.

7 Bayliss EA, Ellis JL, Shoup JA, et al. Effect of continuity of care on hospital utilization for seniors with multiple medical conditions in an integrated health care system. Ann Fam Med 2015;13:123-9.

8 Rizzuto D, Melis RJF, Angleman S, et al. Effect of chronic diseases and multimorbidity on survival and functioning in elderly adults. J Am Geriatr Soc 2017;65:1056-60.

9 Garin N, Olaya B, Moneta MV, et al. Impact of multimorbidity on disability and quality of life in the Spanish older population. PLoS One 2014;9:e11149811.

10 Stairmand J, Gurney J, Stanley J. The impact of multimorbidity on people's lives: a cross-sectional survey. New Zealand Medical Journal 2018;131:78-90.

11 Moffat K, Mercer SW. Challenges of managing people with multimorbidity in today's healthcare systems. BMC Fam Pract 2015;16:1.

12 Hu R-H, Hsiao F-Y, Chen L-J, et al. Increasing age- and genderspecific burden and complexity of multimorbidity in Taiwan, 20032013: a cross-sectional study based on nationwide claims data. BMJ Open 2019;9:e28333.

13 Wang SB, D'Arcy C, Yu YQ, et al. Prevalence and patterns of multimorbidity in northeastern China: a cross-sectional study. Public Health 2015;129:1539-46.

14 Wang HHX, Wang JJ, Wong SYS, et al. Epidemiology of multimorbidity in China and implications for the healthcare system: cross-sectional survey among 162,464 community household residents in southern China. BMC Med 2014;12:188.

15 Prados-Torres A, Calderón-Larrañaga A, Hancco-Saavedra J, et al. Multimorbidity patterns: a systematic review. J Clin Epidemio 2014;67:254-66.

16 Aguado A, Moratalla-Navarro F, López-Simarro F, et al. MorbiNet: multimorbidity networks in adult general population. Analysis of type 2 diabetes mellitus comorbidity. Sci Rep 2020;10:2416.

17 Hidalgo CA, Blumm N, Barabási A-L, et al. A dynamic network approach for the study of human phenotypes. PLoS Comput Biol 2009;5:e1000353.

18 Glicksberg BS, Li L, Badgeley MA, et al. Comparative analyses of population-scale phenomic data in electronic medical records reveal race-specific disease networks. Bioinformatics 2016;32:i101-10.

19 Kalgotra P, Sharda R, Croff JM. Examining health disparities by gender: a multimorbidity network analysis of electronic medical record. Int J Med Inform 2017;108:22-8.

20 Kalgotra P, Sharda R, Croff JM. Examining multimorbidity differences across racial groups: a network analysis of electronic medical records. Sci Rep 2020;10:13538.

21 Harding A. Actor-Network-Theory and Micro-Learning networks. Educ Prim Care 2017;28:295-6.

22 Tang C-L, Wang W-X, Wu X, et al. Effects of average degree on cooperation in networked evolutionary game. Eur Phys $J B$ 2006;53:411-5.

23 Jin L, Guo X, Dou J, et al. Multimorbidity analysis according to sex and age towards cardiovascular diseases of adults in northeast China. Sci Rep 2018;8:8607.

24 Violán C, Roso-Llorach A, Foguet-Boreu Q, et al. Multimorbidity patterns with k-means nonhierarchical cluster analysis. BMC Fam Pract 2018:19:108.

25 Violán C, Foguet-Boreu Q, Fernández-Bertolín S, et al. Soft clustering using real-world data for the identification of multimorbidity patterns in an elderly population: cross-sectional study in a Mediterranean population. BMJ Open 2019;9:e029594.

26 Singer M. A dose of drugs, a touch of violence, a case of AIDS: Conceptualizing the SAVA syndemic. Free Inquiry in Creative Sociology 2000;28:13-24.
27 Schäfer I, von Leitner E-C, Schön G, et al. Multimorbidity patterns in the elderly: a new approach of disease clustering identifies complex interrelations between chronic conditions. PLoS One 2010;5:e1594112.

28 Schäfer I, Kaduszkiewicz H, Wagner $\mathrm{H}-\mathrm{O}$, et al. Reducing complexity: a visualisation of multimorbidity by combining disease clusters and triads. BMC Public Health 2014;14:1285

29 Yu J, Song F, Li Y, et al. Multimorbidity analysis of 13 systemic diseases in northeast China. Int J Environ Res Public Health 2020;17:18176.

30 Muga MA, Owili PO, Hsu C-Y, et al. Association between dietary patterns and cardiovascular risk factors among middle-aged and elderly adults in Taiwan: a population-based study from 2003 to 2012. PLoS One 2016;11:e157745.

31 Yu J, Tao Y, Dou J, et al. The dose-response analysis between $\mathrm{BMI}$ and common chronic diseases in northeast China. Sci Rep 2018;8:4228.

$32 \mathrm{He}$ D, Yu Y, Wu S, et al. Mixed cerebrovascular disease in an elderly patient with mixed vascular risk factors: a case report. BMC Neurol 2019;19:11.

33 Pollack LM, Wang M, Leung MYM, et al. Obesity-Related multimorbidity and risk of cardiovascular disease in the middle-aged population in the United States. Prev Med 2020;139:106225.

34 Taylor LE, Sullivan JC. Sex differences in obesity-induced hypertension and vascular dysfunction: a protective role for estrogen in adipose tissue inflammation? Am J Physiol Regul Integr Comp Physiol 2016;311:R714-20.

35 Shiue I. Self and environmental exposures to drinking, smoking, gambling or video game addiction are associated with adult hypertension, heart and cerebrovascular diseases, allergy, self-rated health and happiness: Japanese General social survey, 2010. Int J Cardiol 2015:181:403-12.

36 Polsky S, Akturk HK. Alcohol consumption, diabetes risk, and cardiovascular disease within diabetes. Curr Diab Rep 2017;17:13612

$37 \mathrm{Yu}$ J, Ma Y, Yang S. Risk factors for cardiovascular disease and their clustering among adults in Jilin (China). Int J Environ Res Public Health 2015;131:h13010070.

38 Salive ME. Multimorbidity in older adults. Epidemiol Rev 2013;35:75-83.

39 Ishizaki T, Kobayashi E, Fukaya T, et al. Association of physical performance and self-rated health with multimorbidity among older adults: results from a nationwide survey in Japan. Arch Gerontol Geriatr 2019;84:103904.

40 Zemedikun DT, Gray LJ, Khunti K, et al. Patterns of multimorbidity in middle-aged and older adults: an analysis of the UK Biobank data. Mayo Clin Proc 2018;93:857-66.

41 Teng $\mathrm{B}$, Zhang $\mathrm{X}, \mathrm{Yi} \mathrm{C}$, et al. The association between ambient air pollution and allergic rhinitis: further epidemiological evidence from Changchun, northeastern China. Int J Environ Res Public Health 2017;14:226.

42 Zheng P-W, Wang J-B, Zhang Z-Y, et al. Air pollution and hospital visits for acute upper and lower respiratory infections among children in Ningbo, China: a time-series analysis. Environ Sci Pollut Res Int 2017;24:18860-9.

$43 \mathrm{Yu} \mathrm{H}$, Huang J, Huai Y, et al. The substantial hospitalization burden of influenza in central China: surveillance for severe, acute respiratory infection, and influenza viruses, 2010-2012. Influenza Other Respir Viruses 2014:8:53-65.

44 Patti AM, Pafili K, Papanas N, et al. Metabolic disorders during pregnancy and postpartum cardiometabolic risk. Endocr Connect 2018;7:E1-4.

45 Specogna AV, Turin TC, Patten SB, et al. Hospital treatment costs and length of stay associated with hypertension and multimorbidity after hemorrhagic stroke. BMC Neurol 2017;17:158. 